\title{
Is preoperative CA-125 level a predictor of cytoreduction in carcinoma ovary?
}

\author{
Premalatha T S ${ }^{1}$, Sumangala G ${ }^{2, *}$, Geeta Acharya ${ }^{3}$, Kiran Kulkarni', Vishakha Bidkar ${ }^{5}$, Pinnaka Vamsi ${ }^{6}$ \\ ${ }^{1}$ Professor and HOD, ${ }^{2,5} \mathrm{MCh}$. Resident, ${ }^{3,4}$ Assistant Professor, ${ }^{5}$ Senior Resident, Dept. of Gynaecologic Oncology, St John's \\ National Academy of Health Sciences, Bengaluru, Karnataka, India \\ *Corresponding Author: Sumangala G \\ Email: sgskp13@gmail.com
}

Received: $9^{\text {th }}$ August, 2018

Accepted: $29^{\text {th }}$ October, 2018

\begin{abstract}
Optimal cyto-reduction of advanced EOC has profound impact on the survival and prognosis of patients3. Suboptimal cytoreduction of advanced ovarian cancer is related to initial tumor bulk which indirectly correlates with serum CA125 levels. Can assessment of level of CA 125 in pre-opererative period predict optimal cyto-reduction is the aim of the study. The mean age of the 70 patients in this study was 49.9 years (SD- 11.75, Range: 21-69 years). Fourty four (63\%) had stage III-IV disease. Sixty five $(93 \%)$ patients had papillary serous ovarian cancer. The serum CA125 levels ranged from 6- $5300 \mathrm{U} / \mathrm{ml}$ with median preoperative serum CA-125 level for all the patients being $259 \mathrm{U} / \mathrm{ml}$ (IQR- 682.98U/ml). The level was elevated (>35 U/ml) in $49 \%$ of patients with stage I-П compared with $93 \%$ of patients with stage $\amalg$ or IV disease. Optimal tumor cyto-reduction was obtained in $61 / 70$ cases $(87 \%)$ of total patients and in 35/44 (80\%) of patients with stage III-IV disease. Diaphragmatic stripping, appendecetomy, recto-sigmoid resection and anastomosis, pelvic peritonectomy, peritoneal stripping/ debulking of peritoneal disease, hemi-colectomy, ileo colic anastomosis and partial nephrectomy. Most of our patients were in intermediate surgical complexity category with a score between 4-7. In 9 patients all belonging to advanced stage, sub-optimal cyto-reduction was done due to multiple co-morbidities, multiple mesenteric lymphnode involvement, multiple small bowel and liver parenchymal deposits and patient not willing for colostomy. An ROC curve analysis showed threshold value of CA125 of $411 \mathrm{U} / \mathrm{ml}$. With a cut-off of $411 \mathrm{U} / \mathrm{ml}, 97 \%$ of total patients with CA125 levels lower than $411 \mathrm{U} / \mathrm{ml}$ were able to undergo optimal cyto-reduction. Also, in $76 \%$ of patients with CA125 level more than $411 \mathrm{U} / \mathrm{ml}$ optimal cyto-reduction could be achieved. When only advanced stages (stage III-IV) were considered, with a cut-off of $411 \mathrm{U} / \mathrm{ml}, 88 \%$ of patients with CA125 levels lower than $411 \mathrm{U} / \mathrm{ml}$ were able to undergo optimal cyto-reduction. Also, in $73 \%$ of patients with CA125 level more than $411 \mathrm{U} / \mathrm{ml}$ optimal cyto-reduction could be achieved. With a pre-operative cut-off value of CA125 more than $411 \mathrm{U} / \mathrm{ml}$, the sensitivity, that is, probability to predict sub-optimal cyto-reduction was $65 \%$ and specificity, that is, probability to predict optimal cyto-reduction was only $67 \%$. Which showed poor predictability for resectability which was in concordance with other studies. Though it is difficult to draw definite conclus
\end{abstract}

Keywords: Carcinoma ovary, CA125, Cyto-reduction, Surgical complexicity score.

\section{Introduction}

Ovarian cancer is the leading cause of death from all Gynecological malignancies. ${ }^{1}$ The standard treatment for advanced epithelial ovarian cancer (EOC), primary carcinoma of fallopian tube and primary peritoneal carcinoma (PPC) is primary debulking surgery (PDS) followed by platinum- and taxane-based chemotherapy. ${ }^{2}$ The aim of the primary surgery is to know the stage of the patient and to resect all grossly visible metastatic disease. Optimal cyto-reduction of advanced EOC has profound impact on the survival and prognosis of patients. ${ }^{3}$ Suboptimal cyto-reduction of advanced ovarian cancer is related to initial tumor bulk which indirectly correlates with serum CA125 level ${ }^{4}$. Serum CA- 125 considered as the gold standard tumor marker in carcinoma ovary and most widely used tumor marker, a monoclonal antibody which detects an antigen present in peripheral blood was discovered by Bast et al in $1983^{3}$ and has been used to assess the tumor burden. In carcinoma ovary, the serum CA- 125 assay has been used in the screening of ovarian cancer, to aid the diagnosis of carcinoma ovary, to monitor the response of treatment delivered and to diagnose recurrence in surveillance period. CA-125 is elevated
(>35 U/ml) in almost 90\% of International Federation of Gynaecology and Obstetrics (FIGO) advanced-stage epithelial ovarian Cancers (Stage III/IV) and less than $50 \%$ of patients with stage I disease. ${ }^{5}$ As serum CA125 is an indirect indicator of tumor burden, can assessment of level of CA 125 in pre-operative period predict optimal cyto-reduction is the aim of the study.

\section{Materials and Methods}

A total of 70 patients with histopathological diagnosis of epithelial ovarian cancer, with preoperative CA 125 value within previous 4 weeks of surgery and operated at St. John's Medical College Hospital were evaluated retrospectively from January 2015 to January 2017 were assessed retrospectively. Exclusion criteria included non epithelial carcinoma ovary, borderline ovarian tumors, epithelial ovarian tumors operated elsewhere, epithelial ovarian tumors which were treated with neo-adjuvant chemotherapy. Medical records were reviewed to collect the demographic details of patients. Demographic details of the patients noted were age, Eastern co-operative oncology group- performance status (ECOG-PS), parity, post-menopausal status, associated co- 
morbidities and body mass index. Pre-operative CA125 assessment was done in all the patients. Serum CA-125 levels were determined by a commercially available radioimmunoassay. All patients had chest x-ray, ultrasound imaging of abdomen and pelvis. Most of the patients also had contrast enhanced CT of abdomen and pelvis as a part of assessment. The operating team in all surgeries included a staff and a resident from the Department of Gynaecologic oncology. Total abdominal hysterectomy with bilateral salpingectomy and infra-colic omentectomy was done in all patients. Peritoneal cytology, peritoneal biopsy and tumor debulking was done as indicated. Intra- operative findings, operative procedure performed, residual disease, site size and number of residual disease at the completion of the procedure and final histopathologic diagnosis was noted. Optimal cyto-reduction was defined as less than $1 \mathrm{~cm}$ of residual disease after cytoreductive surgery in accordance with studies published by the Gynaecologic Oncology Group. ${ }^{6}$ Receiver operating characteristic (ROC) curve was used to determine the CA-125 level with the maximum prognostic power in predicting optimal versus suboptimal debulking.

\section{Results}

The mean age of the 70 patients in this study was 49.9 years (SD- 11.75, Range: 21-69 years). most of our patients $(85 \%)$ presented with abdominal pain associated with abdominal distention. $90 \%$ of the women were parous and $85 \%$ of women were postmenopausal. $50 \%$ of the women had ECOG-PS 1 followed by $37 \%$ of women having ECOG-PS 0 . Diabetes and hypertension were the most commen comorbidities seen in our set of patients. Mean body mass index was 30.7 with a range of 23.7 to 42 . Fourty four (63\%) had stage III-IV, with stage IIIc being the commonest. $26(37 \%)$ patients and stage I-II disease. Sixty five $(93 \%)$ patients had papillary serous and 5 (7\%) patients had non serous tumor histology namely two endometrioid, two clear cell and one mucinous tumor. The serum CA125 levels ranged from 6- 5300 $\mathrm{U} / \mathrm{ml}$ with median pre-operative serum CA-125 level for all the patients being $259 \mathrm{U} / \mathrm{ml}$ (IQR- 682.98U/ml).
The level was elevated (>35 U/ml) in $49 \%$ of patients with stage I-П compared with $93 \%$ of patients with stage $\amalg$ or IV disease. Optimal tumor cyto-reduction was obtained in $61 / 70$ cases $(87 \%)$ of total patients and in 35/44 (80\%) of patients with stage III-IV disease which is shown in table 1 . Surgeries which were done to achieve optimal cytoreduction includedDiaphragmatic stripping, appendecetomy, rectosigmoid resection and anastomosis, pelvic peritonectomy, peritoneal stripping/ debulking of peritoneal disease, hemi-colectomy, ileo colic anastomosis and partial nephrectomy. Most of our patients had intermediate category in surgical complexity scoring system with a score between 4-7. The maximum residual tumor size was defined as the largest diameter of the largest residual tumor lesion. In nine patients all belonging to advanced stage, suboptimal cyto-reduction was done due to multiple comorbidities, multiple mesenteric lymphnode involvement, multiple small bowel and liver parenchymal deposits and patient not willing for colostomy. The largest tumor size noted in this series was $5 \mathrm{~cm}$ liver parenchymal metastases. An ROC curve analysis was done to note the threshold serum CA-125 level below and above which it would probably predict the optimal and sub-optimal cyto-reduction, shown in Fig. 1. The threshold value of CA125 was found to be $411 \mathrm{U} / \mathrm{ml}$. With a cut-off of $411 \mathrm{U} / \mathrm{ml}, 97 \%$ of total patients with CA125 levels lower than $411 \mathrm{U} / \mathrm{ml}$ were able to undergo optimal cyto-reduction. Also, in $76 \%$ of patients with CA125 level more than $411 \mathrm{U} / \mathrm{ml}$ optimal cyto-reduction could be achieved (Table 2 ). When only advanced stages (stage III-IV) were considered, with a cut-off of $411 \mathrm{U} / \mathrm{ml}, 88 \%$ of patients with CA125 levels lower than $411 \mathrm{U} / \mathrm{ml}$ were able to undergo optimal cytoreduction. Also, in $73 \%$ of patients with CA125 level more than $411 \mathrm{U} / \mathrm{ml}$ optimal cyto-reduction could be achieved (Table 3 ). With a pre-operative cut-off value of CA125 more than $411 \mathrm{U} / \mathrm{ml}$, the sensitivity, that is, probability to predict sub-optimal cyto-reduction was $65 \%$ and specificity, that is, probability to predict optimal cyto-reduction was only $67 \%$.

Table 1

\begin{tabular}{|l|c|c|c|c|}
\hline Variable & & $\begin{array}{c}\text { Total patients } \\
(\mathbf{n = 7 0})\end{array}$ & Stage I-II $(\mathbf{n = 2 6})$ & Stage III-IV (n=44) \\
\hline CA125 & Range & $6-5300 \mathrm{U} / \mathrm{ml}$ & $8-5000 \mathrm{U} / \mathrm{ml}$ & $21-5300 \mathrm{U} / \mathrm{ml}$ \\
\hline & mean & $695 \mathrm{U} / \mathrm{ml}$ & $640 \mathrm{U} / \mathrm{ml}$ & $720 \mathrm{Ul} / \mathrm{ml}$ \\
\hline $\begin{array}{l}\text { Optimal tumor } \\
\text { cytoreduction }\end{array}$ & - & $61(87 \%)$ & - & $35(80 \%)$ \\
\hline
\end{tabular}




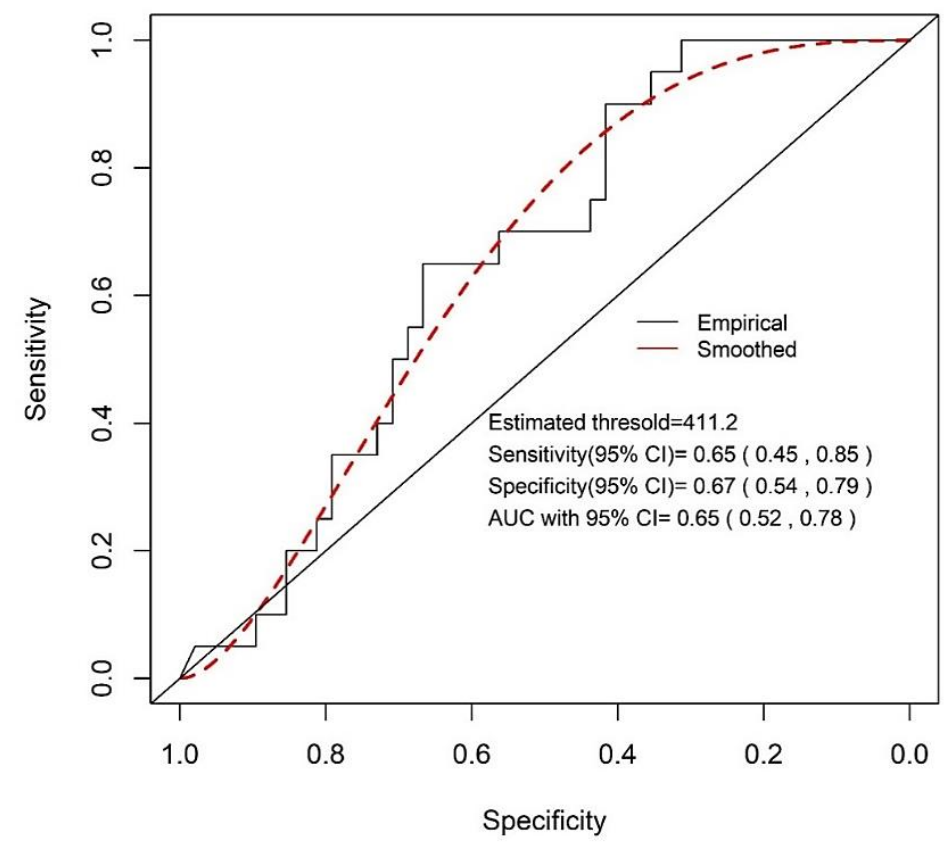

Fig. 1

Table 2: Pre-operative Ca125 level and type of cyto-reduction achieved in total patients $(n=70)$

\begin{tabular}{|l|c|c|c|}
\hline \multicolumn{1}{|c|}{ CA125 levels } & $\mathbf{n}(\boldsymbol{\%})$ & $\begin{array}{c}\text { Optimal cyto- } \\
\text { reduction }\end{array}$ & $\begin{array}{c}\text { Sub-optimal cyto- } \\
\text { reduction }\end{array}$ \\
\hline$>411 \mathrm{U} / \mathrm{ml}$ & $33(47 \%)$ & $25(76 \%)$ & $8(24 \%)$ \\
\hline$<411 \mathrm{U} / \mathrm{ml}$ & $37(53 \%)$ & $36(97 \%)$ & $1(3 \%)$ \\
\hline
\end{tabular}

Table 3: Pre-operative Ca125 level and type of cyto-reduction achieved in stage III-IV (n=44)

\begin{tabular}{|l|c|c|c|}
\hline CA125 levels & n (\%) & Optimal cyto-reduction & $\begin{array}{c}\text { Sub-optimal } \\
\text { cyto-reduction }\end{array}$ \\
\hline$>411 \mathrm{U} / \mathrm{ml}$ & $26(60 \%)$ & $19(73 \%)$ & $7(27 \%)$ \\
\hline$<411 \mathrm{U} / \mathrm{ml}$ & $18(40 \%)$ & $16(88 \%)$ & $2(12 \%)$ \\
\hline
\end{tabular}

\section{Discussion}

Preoperatively to predict accurately and precisiely which patient will have no residual disease status will be at the completion of surgery is difficult. To not do a primary cytoreduction based on findings as determined by imaging or laboratory values means to deviate from the exisisting standard of care to those patients that can be optimally debulked an intervention associated with improved clinical outcomes, most importantly, prolonged overall survival. The role of CA125 levels predicting suboptimal cyto-reduction is still questionable and yet remains controversial. Serum CA 125 assessment is widely available and is of less cost. Though some studies show that preoperative levels may reflect tumor burden, ${ }^{7}$ many studies also have found no such relation. ${ }^{8}$ Laparotomy with optimal debulking has showed better survival in some studies and preoperative level of CA125 is used to predict optimal tumor cytoreduction in advanced ovarian cancer. ${ }^{9}$ Gynecologic Oncology Group defines optimal cytoreduction as residual disease $=1 \mathrm{~cm}$ in maximal diameter. Outcomes are better for patients with no macroscopic disease compared to those with larger implants. Hence, no residual disease should be the surgeon's goal. Investigators at Memorial Sloan-Kettering Cancer Center observed that patients with CA-125 values greater than $500 \mathrm{U} / \mathrm{mL}$ were more likely to have extensive upper abdominal surgery to achieve residual disease status of less than one centimeter, suggesting that CA-125 increases in relation to tumor burden. Detection of threshold preoperative value of serum level of CA-125 for predicting the optimal versus suboptimal debulking is important to decide on the primary modality of treatment- primary debulking surgery versus neo-adjuvant chemotherapy followed by debulking surgery. ${ }^{10}$ High preoperative CA125 serum levels was associated with decreased likelihood of achieving an optimal cyto-reduction in few studies ${ }^{11}$ Contrarily, in other studies preoperative serum CA125 level was not found to be a reliable predictor of optimal cyto-reduction. ${ }^{12}$ In our study, when all seventy patients were considered $97 \%$ of patients with preoperative CA125 levels less than $411 \mathrm{U} / \mathrm{ml}$ underwent optimal debulking, also $76 \%$ of patients with preoperative CA- 
125 levels above $411 \mathrm{U} / \mathrm{ml}$ were also optimally cytoreduced. Even when sub-group analysis of stage $\amalg$ or IV disease was done, $88 \%$ of patients with preoperative CA-125 levels less than $411 \mathrm{U} / \mathrm{ml}$ underwent optimal debulking, also $73 \%$ of patients with levels above $411 \mathrm{U} / \mathrm{ml}$ were also optimally cytoreduced. In this study, both, sensitivity and specificity of preoperative CA- 125 to predict resectability was found to be poor with $65 \%$ and $67 \%$ respectively. The positive and negative likelihood ratios were 1.90 (95\% CI, 1.01- 3.58) and 0.51 (95\% CI, 0.310.86) Similar finding was seen in other studies ${ }^{13}$ were serum CA125 levels were not found to be a good predictor of optimal cyto-reduction.

\section{Conclusion}

Though it is difficult to draw definite conclusions from this study because of small numbers this study suggested that pre-operative CA125 level was not found to be a good predictor of optimal or sub-optimal cyto-reduction. Hence, CA125 alone should not be used to decide on primary debulking surgery especially in small set-ups were sub-optimal cytoreduction is a high probability. CA125 needs to be aided with other features like of clinical findings like massive ascites, performance status and imaging in decision making for proper selection of patient for primary debulking surgery.

\section{References}

1. Siegel R, Ma J, Zou Z. Cancer statistics, 2014. CA Cancer J Clin. 2014;64:9-29.

2. Jing Zeng, Jie Yin, Xiao Song, Ying Jin, Yan Li, and Lingya. Reduction of CA125 Levels during Neoadjuvant Chemotherapy Can Predict Cytoreduction to No Visible Residual Disease in Patients with Advanced Epithelial Ovarian Cancer, Primary Carcinoma of Fallopian tube and Peritoneal Carcinoma. J Cancer. 2016;7(15):2327-2332.

3. M. Modarres-Gilani, F. Ghaemmaghami, S. Ansaripoor, M. Shariat and F. Zaeri. can preoperative ca-125 predict resectability of tumor in patients with advanced epithelial ovarian carcinoma?: Acta Medica Iranica. 2004;42(6).

4. Ofer Gemer, Shmuel Segal \& Alexander Kopmar. Preoperative CA-125 level as a predictor of non optimal cytoreduction of advanced epithelial ovarian cancer. $p$. 583-585.

5. Jacobs I, Bast RC Jr. The CA 125 tumour-associated antigen: a review of the literature. Hum Reprod. 1989;4(1):1-12.

6. Hoskins WJ, Bundy BN, Thigpen JT, Omura GA. The influence of cytoreductive surgery on recurrence-free interval and survival in small-volume stage III epithelial ovarian cancer: a Gynecologic Oncology Group study. Gynecol Oncol. 1992;47(2):159-166.

7. Bast RC, Jr, Urban N, Shridhar V. Early detection of ovarian cancer: promise and reality. Cancer Treat Res. 2002;107:61-97.

8. Kumar P, Rehani MM, Kumar L. Tumor marker CA-125 as an evaluator and response indicator in ovarian cancer: its quantitative correlation with tumor volume. Med Sci Monit. 2005;11:CR84-9.
9. Mury D, Woelber L, Jung S. Prognostic and predictive relevance of CA-125 at primary surgery of ovarian cancer. J Cancer Res Clin Oncol. 2011;1377:1131-7.

10. Chi DS, Venkatraman ES, Masson V, Hoskins WJ. The ability of preoperative serum CA-125 to predict optimal primary tumor cytoreduction in stage III epithelial ovarian carcinoma. Gynecol Oncol. 2000;77(2):227-231.

11. Gemer O, Lurian M, Gdalevich M. A multicenter study of CA 125 level as a predictor of non-optimal primary cytoreduction of advanced epithelial ovarian cancer. Europ J Surg Oncol. 2005;319:1006-10.

12. Kang S, Kim TJ, Nam BH. Preoperative serum CA- 125 levels and risk of suboptimal cytoreduction in ovarian cancer: a meta analysis. J Surg Oncol. 2010;1011:13-17.

13. Cooper BC, Sood AK, Davis CS, Ritchie JM, Sorosky JI, Anderson B, Buller RE. Preoperative CA 125 levels: an independent prognostic factor for epithelial ovarian cancer. Obstet Gynecol. 2002;100(1):59-64.

How to cite this article: Premalatha $\mathrm{T} S$, Sumangala G, Acharya G, Kulkarni K, Bidkar V, Vamsi P. Is preoperative CA-125 level a predictor of cytoreduction in carcinoma ovary? Indian $\mathrm{J}$ Obstet Gynecol Res. 2018;5(4):516-519. 